\title{
Auditory-vocal integration impairment: New challenges and opportunities for voice assessment and therapy
}

\section{Alteración de la integración auditivo-vocal: nuevos retos y oportunidades para la evaluación y la terapia de la voz}

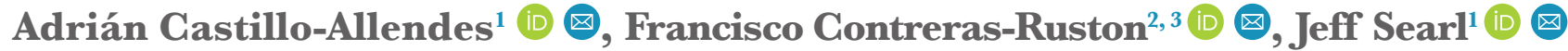 \\ ${ }^{1}$ Department of Communicative Sciences and Disorders; Michigan State University; East Lansing; United States. \\ ${ }^{2}$ Perception, Attention and Representation Lab; Department of Cognition, Development and Educational Psychology; Universidad de \\ Barcelona; Barcelona; España.
}

${ }^{3}$ Departamento de Fonoaudiología; Universidad de Valparaíso; Valparaíso; Chile.

Correspondence

Adrián Castillo-Allendes.

Email: casti208@msu.edu

\section{How to cite \\ Castillo-Allendes, Adrián; Contreras-Ruston, Francisco; Searl, Jeff. (2021). Auditory-vocal integration impairment: New challenges and opportunities for voice assessment and therapy. Revista de Investigación e Innovación en Ciencias de la Salud. 3(2), 87-97. https://doi.org/10.46634/riics.62}

Received: $26 / 04 / 2021$

Revised: 28/09/2021

Accepted: 20/11/2021

\section{Invited editor}

Carlos Manzano Aquiahuatl, MD, MSc.

\section{Editor en jefe}

Jorge Mauricio Cuartas Arias, Ph.D. (iD

\section{Coeditor}

Fraidy-Alonso Alzate-Pamplona, MSc. ID

Gopyright $\odot$ 2021. Fundación Universitaria María Cano. The Revista de Investigación e Innovación en Ciencias de la Salud provides open access to all its content under the terms of the Creative Commons Attribution-

NonCommercial-NoDerivatives 4.0

International (CG BY-NG-ND 4.0).

Conflicts of Interest

The authors have declared that no competing interests exist.

\begin{abstract}
This reflection paper addresses the importance of the interaction between voice perception and voice production, emphasizing the processes of auditory-vocal integration that are not yet widely reported in the context of voice clinicians. Given the above, this article seeks to 1) highlight the important link between voice production and voice perception and 2) consider whether this relationship might be exploited clinically for diagnostic purposes and therapeutic benefit. Existing theories on speech production and its interaction with auditory perception provide context for discussing why the evaluation of auditory-vocal processes could help identify associated origins of dysphonia and inform the clinician around appropriate management strategies. Incorporating auditory-vocal integration assessment through sensorimotor adaptation paradigm testing could prove to be an important addition to voice assessment protocols at the clinical level. Further, if future studies can specify the means to manipulate and enhance a person's auditory-vocal integration, the efficiency of voice therapy could be increased, leading to improved quality of life for people with voice disorders.
\end{abstract}

\section{Keywords}

Voice disorders; dysphonia, auditory feedback; auditory-vocal impairment; motor control; voice assessment; voice therapy; DIVA model; altered feedback; sensorimotor processing; SimpleDIVA model.

\section{Resumen}

Este artículo de reflexión aborda la importancia de la interacción entre la percepción y la producción de la voz, haciendo hincapié en los procesos de integración auditi- 
Data Availability Statement

All relevant data is in the article and in the appendices. For more detailed information, write to the Corresponding Author.

\section{Funding}

None. This research did not receive any specific grants from funding agencies in the public, commercial, or non-profit sectors.

\section{Disclaimer}

The content of this article is the sole responsibility of the authors and does not represent an official opinion of their institutions or the Revista de Investigación e Innovación en Ciencias de la Salud.

\section{Acknowledgments}

The first author would like to thank Dr. Laura Dilley for introducing him to the world of speech perception and allowing him to explore its relationship to voice production and disorders.

\section{Author Contributions} Adrián Castillo-Allendes:

conceptualization, investigation, project administration, writing - original draft, writing - review and editing: Francisco Contreras-Ruston: conceptualization, resources, writing original draft, writing - review and editing. Jeff Searl: conceptualization, resources, supervision, writing - review and editing. vo-vocal, los cuales aún no han sido muy divulgados en el contexto de los clínicos de voz. Dado lo anterior, este articulo busca: 1) destacar la importante relación entre la producción y la percepción de la voz y 2) considerar si esta relación pudiese explotarse clínicamente con fines diagnósticos y terapéuticos. Las teorías existentes sobre la producción de la voz y su interacción con la percepción auditiva proporcionan el contexto para discutir por qué la evaluación de los procesos auditivo-vocales podría ayudar a identificar los orígenes asociados a cierto tipo de disfonías e informar al clínico sobre las estrategias de abordaje adecuadas. La incorporación de la evaluación de la integración auditivo-vocal a través de la prueba del paradigma de adaptación sensoriomotora podría ser una importante adición a los protocolos de evaluación de la voz a nivel clínico. Además, si los estudios futuros pueden especificar los medios para manipular y mejorar la integración auditivo-vocal de una persona, la eficacia de la terapia de la voz podría aumentar, lo que llevaría a mejorar la calidad de vida de las personas con trastornos de la voz.

\section{Palabras clave}

Trastornos de la voz; disfonía, retroalimentación auditiva; alteración auditivo-vocal; control motor; evaluación de la voz; terapia de la voz; modelo DIVA; retroalimentación alterada; procesamiento sensoriomotor; modelo SimpleDIVA.

\section{Introduction}

Human communication is a complex process that can be considered from various perspectives. It depends on several factors, one of which is the interaction between speech perception and speech production. The perceptual-motor relationship is inextricably integrated not only in speech tasks but also in perception, auditory system, cognition, and language, among others [1-3]. One of the critical aspects of motor speech production is phonation, which plays an essential role in the listener's speech perception and auditory self-voice feedback received as an individual speaks [4].

From the listeners' perspective, voiced sound carries selective spectral modification comprised from articulatory gestures of the vocal tract, resulting in a signal containing harmonic energy in a wide range of frequencies, covering at least the vocal tract's first acoustic resonances [5]. Furthermore, vowel formant frequencies and transitions (change in formant frequency of a vowel immediately before or after a consonant) can affect the interpretation of vowels and the adjacent consonant, generating the perception of a word [6-11].

Regarding the speaker, auditory self-voice feedback plays a unique role. Once vocalization is initiated, auditory feedback monitors possible acoustic changes that may occur during speech, allowing control over the speaker's vocal and articulatory output $[12,13]$. The above are examples of the inherent interaction between speech production and speech perception in both speaker and listener. Nonetheless, this phenomenon of interaction between auditory feedback and voice has not yet been incorporated into the routine clinical evaluation of voice problems.

In general terms, a comprehensive voice assessment is based on information from numerous sources, including acoustics, aerodynamics, endoscopy of the larynx, clinical judgment of vocal quality, and the patient's self-perception of their voice in terms of its quality and impact on their life [14]. Just in recent years, the important role of 
auditory feedback in voice production has begun to be described for both voice assessment and voice therapy; however, some aspects related to auditory-vocal integration are still not widely known by voice clinicians. Because of the above, this reflection article has two purposes: 1) to highlight the important link between voice production and voice perception and 2) to consider whether this relationship might be exploited clinically for diagnostic purposes and therapeutic benefit. Existing theories on speech production and its interaction with auditory perception provide context for discussing why the evaluation of auditory-vocal processes could help identify associated origins of dysphonia and inform the clinician around appropriate management strategies.

\section{Speech Perception and Production}

Voicing is the primary outcome of the process of speech production. Spoken utterances are then perceived by both the listener and the speaker. The role of auditory input on speech and voice production can be considered from at least two perspectives. First, it is recognized that auditory signals external to the speaker impact how a person produces their voice. The Lombard effect is an example of an external auditory signal that can cause individuals to increase their loudness involuntarily [15]. Also, the Lombard effect causes acoustic and phonetic modifications, including an increase in the fundamental frequency $\left(f_{\mathrm{o}}\right)$, a change in the first $\left(F_{I}\right)$ and second formants $\left(F_{2}\right)$, and an increase in vowel duration [16-18]. Research involving people with Parkinson's disease (PD) has shown that the Lombard effect could even positively affect voice therapy. By altering the subjects' auditory feedback, the person with PD increased their $f_{\mathrm{o}}$, voice intensity, and stability [19]. A second type of auditory input occurs when a speaker perceives their own voice in near-real-time (self-voice feedback). It is this type of feedback that we are most closely considering within this reflection.

Dating back several decades, researchers have conducted auditory-feedback perturbation studies, shedding light on the role of auditory feedback on voice control. In general, auditory perturbation studies involve altering some aspect of the acoustic signal (e.g., vowel formant) that a person is producing and presenting this in near real-time to the same speaker to see if or how they adjust the speech production [20]. As an example, research has verified that when the $f_{\mathrm{o}}$ of one's own voice is modified and presented to the individual during or before vocalization, a compensatory response engages, in which the person adjusts the intended target $f_{\mathrm{o}}$ to match the $f_{\mathrm{o}}$-adjusted stimulus, evidencing that $f_{\mathrm{o}}$ responds dynamically to auditory self-voice feedback [21].

Classical models of speech and language production incorporate perception and production as components in their structural features, such as the Broca-Wernicke-Lichtheim model [22]. In recent decades, scientific evidence, based on neuroimaging studies, has been collected. This evidence shows a cortical and subcortical connection related to the self-perception of speech, processing, and language production [23]. New neurocomputational models incorporate interactive networks, or streams, in their structure, allowing for a better understanding of the interaction between perception and speech production and relating comprehension and production processes to ventral and dorsal regions of the brain [24,25].

We can mention models that attempt to explain this phenomenon, to relate some of their components to voice control and production: the Directions Into Velocities of Articulators (DIVA), the State Feedback Controller (SFG), and recently the SimpleDIVA models [24,26,27]. 
The DIVA and SFG models are neurocomputational models to describe the speech production process [24]. These models emphasize the role of auditory and sensorimotor feedback in planning speech motor responses. In addition, these incorporate anatomical labels of different brain regions at each stage, or map, which are connected by synaptic projection $[24,28,29]$. Specifically, the DIVA and SFC models' systems both include auditory feedback that allows detection of speech errors, so that corrections can be attempted and a desired speech motor response is generated [29,30]. The model encodes movement velocities for the lips, tongue, jaw, and larynx $[24,28,29]$. The difference is that DIVA relies primarily on feedforward controls, whereas SCF integrates internal predictions through efference copies, allowing for an increased gain during vocalization [26,31].

We focus on the recent proposal of Kearney and colleagues who described a simplified version of the DIVA model, the SimpleDIVA, for being specific in voice self-feedback [27]. This model is a three-parameter mathematical model that quantifies the associated three subsystems involved in speech control: auditory feedback control, somatosensory feedback control, and feedforward control mechanisms to sensorimotor adaptation. In this model, the feedforward controller consists of stored motor sequences updated based on sensory errors. Detection of sensory errors occurs from an auditory feedback control component in the model that essentially compares the planned motor speech output from the feedforward controller with the speaker's auditory signal. Similarly, a somatosensory feedback control component is part of the model engaged when somatosensory feedback from articulators detects errors compared to the planned motor output. In this manner, sensory feedback is used to make nearreal-time adjustments to output via error detection. SimpleDIVA offers a new understanding of speech and voice control, through a phenomenological explanation for the behavioral responses to the adaptation paradigm challenging to interpret from behavioral data alone. As the authors state, the SimpleDIVA can better understand sensorimotor learning and control differences between normal and disordered groups of speakers, which could ultimately identify new or more refined interventions for those with communication disorders [27].

Contemporary speech motor control models include components within their structure that help explain different types of auditory-vocal disorders and the relationship to auditory integration, evidencing underlying mechanisms of sensorimotor-based communication disorders. A valuable means of studying these issues involves a sensorimotor adaptation paradigm [27]. In this paradigm, a perturbation of the speaker's auditory feedback is created through the modification in real-time of formants or $f_{\mathrm{o}}$ of the speaker during the repeated production of a series of words. Generally, auditory feedback is altered in three different ways during the repetition of utterances after baseline recordings, without auditory feedback manipulation: "ramp" in which a parameter is shifted incrementally over time; "full-shift" or "hold", where a parameter is abruptly altered and held for a time; and "post-shift" or "after-effect", where the alteration is removed $[28,32,33]$.

\section{Evidence of Auditory-vocal Impairment and Behavioral Voice Disorders}

Different classification systems have been proposed for voice disorders. Still, one common designation is the division into functional and organic disorders, with subcategories for functional voice disorders, often referred to as hypofunctional and hyperfunctional disorders. The hyperfunctional category relates to laryngeal muscle strain and ineffective or inadequate phonatory behavior [34]. Hyperfunctional voice disorders are common and have been associated 
with other diagnoses, such as phonoatruamatic and benign lesions of the free edge of the vocal folds [35]. While studies identify a wide range of clinical symptoms and biomechanical and laryngeal configurations, there is an incomplete understanding of the cause(s) of hyperfunctional voice disorders, which have been linked to poor vocal hygiene, aberrant or excessive use of the voice, and psychological and personality factors [32]. Stepp and colleagues, who evaluated auditory-vocal integration impairment in people with diagnosed hyperfunctional voice disorders, hypothesized that such impairment may contribute to developing and maintaining these behavioral voice disorders $[32,36]$. This hypothesis arose from observing the auditory-vocal integration impairment in subjects with hearing loss. Individuals with hearing loss have some voice characteristics similar to those with hyperfunctional dysphonias such as high glottal resistance, increased phonatory effort, and voice quality changes like strain and breathiness $[32,37]$.

In normal conditions of auditory-vocal integration, by exposing a person to an increase in their own $f_{\mathrm{o}}$ (feedback), the expected adaptative response is a decrease in the $f_{\mathrm{o}}$ of the subject's own voice, that is, subjects shift their pitch in the opposite direction to the auditory stimulus as a compensatory response [33]. The brain seeks to predict and recapitulate representations that best adapt to external stimuli and sources, creating advanced predictive models with sensory information to minimize error relative to the intended production [38]. Adaptive responses are influenced by interactions between the feedforward and feedback control systems and are seen when feedback is consistently perturbed [30,39].

Utilizing the sensorimotor adaptation paradigm, Stepp found that the subjects with hyperfunctional voice disorders did not show a typical adaptative response, i.e., when $f_{\mathrm{o}}$ increased, speakers responded by further increasing their $f_{\mathrm{o}}$. The authors interpreted these results as evidence that some people with voice disorder have an auditory-vocal integration impairment, resulting from a deficit between feedforward voice control and auditory feedback. Thus, the presence of auditory-vocal disorder could explain the occurrence and persistence over time of hyperfunctional vocal behaviors [32], an aspect that the SimpleDIVA model could also explain, regarding a deficit in the correction and adaptation of ongoing vocal production due to errors in auditory feedback [27].

Stepp's study contributes to understanding how, for example, an initial change in a person's voice, after phonotraumatic behaviors or an infection of the upper airway, may result in prolonged changes in voice production that can become chronic. An altered voice quality received as feedback from an individuals' own voice, continually altering the feedforward responses of the system, could also help explain why some interventions are not successful for specific individuals. A recent article focused on people with benign vocal fold lesions also implicated auditory-vocal feedback impairments as a factor in developing that specific voice disorder [40]. Lee's study incorporated a group of participants with nodules, polyps, and cysts of the vocal folds and non-dysphonic subjects. The participants were asked to produce a sustained vowel under different auditory feedback conditions in real-time. Unlike the classic sensory adaptation experiment, the auditory feedback modifications consisted of integrating a background noise and enhanced feedback of a self-produced voice. Lee et al. found that low-frequency modulations (below $3 \mathrm{~Hz}$ ) of vocal $f_{\mathrm{o}}$ of a sustained vowel were significantly high for subjects with vocal fold nodules over the other groups. The authors interpreted the results as supportive of the possibility that vocal fold nodules and their vocal behaviors may be associated with abnormal auditory-vocal feedback integration [40]. 


\section{Improving Auditory Feedback as a Therapeutic Approach.}

Emerging literature regarding altered sensorimotor integration relating to voice production suggests an intriguing possibility: targeting auditory-vocal feedback control processes might be a helpful component of therapeutic interventions, which means voice production might be improved, in part, through manipulation of sensory mechanisms or auditory-feedback $[40,41]$. However, as auditory-vocal integration is just beginning to be studied as a possible cause and prevalence of some types of dysphonia, there are still many research questions to be addressed regarding the evaluation and treatment of voice impairment.

Some authors have already started exploring the impact of devices and other interventions to alter or improve auditory feedback on the voice. A recently published study addressed whether auditory feedback control of vocal pitch production in subjects with PD could benefit from Lee Silverman voice treatment (LSVT® LOUD) [42]. LSVT LOUD is an intensive voice treatment program that aims to increase voice intensity in people with hypokinetic dysarthria through a sensorimotor recalibration of increased vocal loudness $[43,44]$. Li's study demonstrated the positive effects of LSVT LOUD on auditory-vocal integration in people with PD [42]. After LSVT LOUD, subjects showed compensatory responses to auditory feedback similar to the performance of healthy subjects. Additionally, significantly greater EEG cortical responses (P2) were observed in response to pitch perturbations after LSVT LOUD, reflecting the intervention's possible top-down modulatory effect on auditory-motor integration for voice regulation in the PD subjects [42].

In addition, it is important to mention that it has been shown that the learning of speech motor sequences is not only based in areas of the brain classically related to learning, but also in those associated with auditory and somatosensory feedback-based speech motor learning and the network of brain regions that participate in both motor and sensory processes [45]. All the above leads us to wonder whether the intensive nature of some voice therapies with a high number of vocal motor task repetitions and consistently used stimuli could conceivably improve feedforward phonatory performance, which could also be explained by the DIVA and SimpleDIVA model.

Other tools that could favor therapeutic use of auditory feedback include, for example, the Escera-assessed device called Forbrain ${ }^{\circledR}$ (Sound For Life Ltd/Soundev, Luxemburg, model UN38.3) as an Auditory Altered Feedback (AAF) device by evaluating changes in voice quality-related acoustic measures such as smoothed cepstral peak prominence (CPPS) and longterm average spectrum (LTAS) [41]. The device allows the users to have real-time improved auditory feedback through bone conduction and amplification of the high or low speech frequencies. The results indicated that the Forbrain ${ }^{\circledR}$ altered the voice signal in the manner described by the manufacturer. However, the AAF feedback had some paradoxical results. The values of the trendline of the LTAS were consistent with improved voice quality. Still, the values of the CPPS, a measure associated with voice quality, decreased, which is associated with worsened quality. The author states that this effect may be due to a typical response to AAF devices, where motor feedforward is altered as a consequence of motor adaptation to improve auditory feedback; conversely, motor output is more accurately adjusted when there is altered feedback. These results can be taken as a research opportunity to test this kind of device by setting different types of auditory feedback perturbation. If beneficial, these tools could be of great utility for voice rehabilitation processes and research due to their ease of implementation and design, which allows performing ecological studies. 
Another interesting fact related to tools that modify auditory feedback is an additional result of Lee's study mentioned above. Bone conduction feedback of the self-produced voice significantly reduced the low-frequency modulations of vocal $f_{\mathrm{o}}$ of a sustained vowel. From this result, the authors raise the reasonability of such an auditory feedback aid being incorporated as a therapeutic modality for vocal folds nodules [40]. Following the same dynamic, these studies in subjects with voice disorders and auditory-vocal impairment could be an excellent opportunity to assess this type of device's usefulness in a population that would probably benefit the most.

\section{Final Considerations}

The production of voice and speech is a complex process that requires interaction with auditory perception. Sensorimotor adaptation provides another avenue to consider in understanding and treating individuals with voice disorders: assessing and manipulating a person's capabilities relative to vocal motor control. Comprehending key and current aspects linked to speech perception and its disorders opens the door to a broader view on understanding the process of human voice production. It is beneficial for a voice researcher and clinician to advance knowledge of the neurobiological mechanisms that support speech and voice perception and how production is shaped by sensory experience (i.e., auditory and somatosensory). This understanding can lead to novel ways to assess and treat a person who has a voice disorder. Therefore, understanding voice production requires an integrated approach [5], where physiology, acoustics, biomechanics, and neurological processes must be considered holistically and not in isolation. Part of an integrated approach involves determining how voice self-perception and production are related.

Emerging work establishing that auditory-vocal impairment is often present in those with functional dysphonia is an important step that may eventually influence the diagnostic and therapeutic voice practice $[32,40]$. An impairment in auditory perception could impact feedforward processes of voicing and subsequently impact the recovery process after acute dysphonia. Incorporating auditory-vocal integration assessment through sensorimotor adaptation paradigm testing could eventually prove to be an important addition to voice evaluation protocols. Further, suppose areas of improvement within a person's auditory-vocal integration can be identified. In that case, voice therapy efficacy and efficiency could be increased, leading to improved quality of life and possibly reduced health-related costs. Moreover, DIVA models suggest that motor output changes may become more long-term by persisting on the integration of auditory feedback within voice therapy. One of the challenges for the future is to take advantage of such information and consider how auditory and somatosensory feedback modifications in subjects with auditory-vocal impairments can be assessed and manipulated. These models can provide important information about the complex and multifactorial nature of the voice production process, which clearly is linked to a person's auditory and somatosensory voice perception.

There are still many challenges about the relationship between voice and auditory feedback. An alteration in the integration between auditory feedback and voice production appears to be a potentially important issue for some people who have a voice disorder. However, the best ways to identify and characterize how a person's auditory-vocal integration is impaired have not been developed to a point where application within a clinical setting can be applied. Similarly, the best approaches to modify and improve a person's auditory-vocal integration capabilities remain to be developed. 


\section{References}

1. Liberman AM, Mattingly IG. The motor theory of speech perception revised. Cognition [nternet]. 1985 Oct;21(1):1-36. doi: https://doi.org/10.1016/0010-0277(85)90021-6

2. Fowler CA. Speech Perception as a Perceptuo-Motor Skill. In: Hickok G, Small S, editors. Neurobiology of Language [Internet]. Elsevier; 2016. p. 175-84. doi: https://doi. org/10.1016/B978-0-12-407794-2.00015-8

3. Lotto AJ, Holt LL. Speech Perception. In: Hickok G, Small S, editors. Neurobiology of Language [Internet]. Elsevier; 2016 [cited 2021 Mar 9]. p. 185-94. doi: https://doi. org/10.1016/B978-0-12-407794-2.00016-X

4. KuangJ, Liberman M. Integrating Voice Quality Cues in the Pitch Perception of Speech and Non-speech Utterances. Front Psychol [Internet]. 2018 Nov 29;9. doi: https://doi. org/10.3389/fpsyg.2018.02147

5. Zhang Z. Mechanics of human voice production and control. J Acoust Soc Am [nternet]. 2016 Oct;140(4):2614-35. doi: https://doi.org/10.1121/1.4964509

6. Liberman AM, Delattre P, Cooper FS. The Role of Selected Stimulus-Variables in the Perception of the Unvoiced Stop Consonants. Am J Psychol [Internet]. 1952 Oct;65(4):497. doi: https://doi.org/10.2307/1418032

7. Liberman AM, Delattre PC, Gerstman LJ, Cooper FS. Tempo of frequency change as a cue for distinguishing classes of speech sounds. J Exp Psychol [Internet]. 1956;52(2):12737. doi: http://doi.apa.org/getdoi.cfm?doi=10.1037/h0041240

8. Liberman AM, Harris KS, Hoffman HS, Griffith BC. The discrimination of speech sounds within and across phoneme boundaries. J Exp Psychol [Internet]. 1957;54(5):35868. doi: http://doi.apa.org/getdoi.cfm?doi=10.1037/h0044417

9. Hillenbrand JM, Houde RA. Role of Fo and Amplitude in the Perception of Intervocalic Glottal Stops. J Speech, Lang Hear Res [Internet]. 1996 Dec;39(6):1 182-90. doi: https://doi.org/10.1044/jshr.3906.1182

10. Hillenbrand J, Getty LA, Clark MJ, Wheeler K. Acoustic characteristics of American English vowels. J Acoust Soc Am [nternet]. 1995 May;97(5):3099-111. doi: https://doi. org/10.1121/1.411872

11. Morrison GS, Assmann PF. Vowel Inherent Spectral Change [Internet]. Morrison GS, Assmann PF, editors. Vowel Inherent Spectral Change. Berlin, Heidelberg: Springer Berlin Heidelberg; 2013. p. 1-286. Available from: http://link.springer.com/10.1007/9783-642-14209-3

12. Patel S, Nishimura C, Lodhavia A, Korzyukov O, Parkinson A, Robin DA, et al. Understanding the mechanisms underlying voluntary responses to pitch-shifted auditory feedback. J Acoust Soc Am [Internet]. 2014 May;135(5):3036-44. doi: https://doi. org/10.1121/1.4870490

13. Parkinson AL, Flagmeier SG, Manes JL, Larson CR, Rogers B, Robin DA. Understanding the neural mechanisms involved in sensory control of voice production. Neuroimage [Internet]. 2012 May;61(1):314-22. doi: https://doi.org/10.1016/j.neuroimage.2012.02.068 
14. Patel RR, Awan SN, Barkmeier-Kraemer J, Courey M, Deliyski D, Eadie T, et al. Recommended protocols for instrumental assessment of voice: American speech-language-hearing association expert panel to develop a protocol for instrumental assessment of vocal function. Am J Speech-Language Pathol [Internet]. 2018 Aug 1 [cited 2021 Mar 9];27(3):887-905. doi: https://doi.org/10.1044/2018_AJSLP-17-0009

15. Whittico TH, Ortiz AJ, Marks KL, Toles LE, Van Stan JH, Hillman RE, et al. Ambulatory monitoring of Lombard-related vocal characteristics in vocally healthy female speakers. J Acoust Soc Am [Internet]. 2020 Jun;147(6):EL552-8. doi: https://doi. org/10.1121/10.0001446

16. Junqua J. The Lombard reflex and its role on human listeners and automatic speech recognizers. J Acoust Soc Am [Internet]. 1993 Jan;93(1):510-24. doi: https://doi. org/10.1121/1.405631

17. Lu Y, Cooke M. Speech production modifications produced by competing talkers, babble, and stationary noise. J Acoust Soc Am [Internet]. 2008 Nov;124(5):3261-75. doi: https://doi.org/10.1121/1.2990705

18. Alghamdi N, Maddock S, Marxer R, Barker J, Brown GJ. A corpus of audio-visual Lombard speech with frontal and profile views. J Acoust Soc Am [Internet]. 2018 Jun;143(6):EL523-9. doi: https://doi.org/10.1121/1.5042758

19. Quedas A, de Campos Duprat A, Gasparini G. Lombard's effect's implication in intensity, fundamental frequency and stability on the voice of individuals with Parkinson's disease. Braz J Otorhinolaryngol [Internet]. 2007 Sep;73(5):675-83. doi: https://doi. org/10.1016/S1808-8694(15)30129-4

20. Purcell DW, Munhall KG. Compensation following real-time manipulation of formants in isolated vowels. J Acoust Soc Am [nternet]. 2006 Apr;119(4):2288-97. doi: https:// doi.org/10.1121/1.2173514

21. Larson GR, Burnett TA, Bauer JJ, Kiran S, Hain TG. Comparison of voice Fo responses to pitch-shift onset and offset conditions. J Acoust Soc Am [Internet]. 2001 Dec;110(6):2845-8. doi: https://doi.org/10.1121/1.1417527

22. Tremblay P, Dick AS. Broca and Wernicke are dead, or moving past the classic model of language neurobiology. Brain Lang [Internet]. 2016 Nov;162:60-71. doi: https://doi. org/10.1016/j.bandl.2016.08.004

23. Dick AS, Bernal B, Tremblay P. The Language Connectome. Neurosci [Internet]. 2014 Oct 15;20(5):453-67. doi: https://doi.org/10.1177/1073858413513502

24. Guenther FH, Hickok G. Role of the auditory system in speech production. In: Aminoff M, Boller F, Swaab D, editora. Handbook of Clinical Neurology. Elsevier B.V.; 2015. p. 161-75. doi: https://doi.org/10.1016/B978-0-444-62630-1.00009-3

25. Nasios G, Dardiotis E, Messinis L. From Broca and Wernicke to the Neuromodulation Era: Insights of Brain Language Networks for Neurorehabilitation. Behav Neurol [nternet]. 2019 Jul 22;2019:1-10. doi: https://doi.org/10.1155/2019/9894571 
26. Hickok G. Computational neuroanatomy of speech production. Nat Rev Neurosci [Internet]. 2012 Feb 5;13(2):135-45. doi: https://doi.org/10.1038/nrn3158

27. Kearney E, Nieto-Castañón A, Weerathunge HR, Falsini R, Daliri A, Abur D, et al. A Simple 3-Parameter Model for Examining Adaptation in Speech and Voice Production. Front Psychol [nternet]. 2020 Jan 21;10. doi: https://doi.org/10.3389/fpsyg.2019.02995

28. Perkell JS. Movement goals and feedback and feedforward control mechanisms in speech production. J Neurolinguistics [Internet]. 2012 Sep;25(5):382-407. doi: https://doi. org/10.1016/j.jneuroling.2010.02.011

29. Tourville JA, Guenther FH. The DIVA model: A neural theory of speech acquisition and production. Lang Cogn Process [Internet]. 2011 Aug;26(7):952-81. doi: https://doi. org/10.1080/01690960903498424

30. Abur D, Lester-Smith RA, Daliri A, Lupiani AA, Guenther FH, Stepp CE. Sensorimotor adaptation of voice fundamental frequency in Parkinson's disease. PLoS One [Internet]. 2018 Jan 26;13(1):e0191839. doi: https://doi.org/10.1371/journal.pone.0191839

31. Houde JF, Nagarajan SS. Speech Production as State Feedback Control. Front Hum Neurosci [Internet]. 2011;5. doi: https://doi.org/10.3389/fnhum.2011.00082

32. Stepp CE, Lester-Smith RA, Abur D, Daliri A, Pieter Noordzij J, Lupiani AA. Evidence for auditory-motor impairment in individuals with hyperfunctional voice disorders [Internet]. Journal of Speech, Language, and Hearing Research. American Speech-Language-Hearing Association; 2017 [cited 2021 Mar 10];60(6):1545-50. doi: https://doi. org/10.1044/2017_JSLHR-S-16-0282

33. Jones JA, Munhall KG. Perceptual calibration of F0 production: Evidence from feedback perturbation. J Acoust Soc Am [Internet]. 2000 [cited 2021 Mar 9];108(3):1246. doi: https://doi.org/10.1121/1.1288414

34. Aronson AE. Clinical Voice Disorders (3rd Ed). Thieme; 1990.

35. Galindo GE, Peterson SD, Erath BD, Gastro C, Hillman RE, Zañartu M. Modeling the Pathophysiology of Phonotraumatic Vocal Hyperfunction With a Triangular Glottal Model of the Vocal Folds. J Speech, Lang Hear Res [Internet]. 2017 Sep 18;60(9):245271. doi: https://doi.org/10.1044/2017_JSLHR-S-16-0412

36. Weerathunge HR, Abur D, Enos NM, Brown KM, Stepp CE. Auditory-Motor Perturbations of Voice Fundamental Frequency: Feedback Delay and Amplification.J Speech, Lang Hear Res [Internet]. 2020 Sep 15;63(9):2846-60. doi: https://doi.org/10.1044/2020_ JSLHR-19-00407

37. Arends N, Povel DJ, Van Os E, Speth L. Predicting voice quality of deaf speakers on the basis of glottal characteristics. J Speech Hear Res [Internet]. 1990 [cited 2021 Mar 9];33(1):116-22. doi: https://doi.org/10.1044/jshr.3301.116

38. Clark A. Whatever next? Predictive brains, situated agents, and the future of cognitive science. Behav Brain Sci [Internet]. 2013 Jun 10 [cited 2021 Mar 9];36(3):181-204. doi: https://doi.org/10.1017/S0140525X12000477 
39. Lester-Smith RA, Daliri A, Enos N, Abur D, Lupiani AA, Letcher S, et al. The relation of articulatory and vocal auditory-motor control in typical speakers. J Speech, Lang Hear Res [Internet]. 2020 Nov 1 [cited 2021 Mar 9];63(11):3628-42. doi: https://doi. org/10.1044/2020_JSLHR-20-00192

40. Lee SH, Yu JF, Fang TJ, Lee GS. Vocal fold nodules: A disorder of phonation organs or auditory feedback? Clin Otolaryngol [Internet]. 2019 Nov 1 [cited 2021 Mar 9];44(6):975-82. doi: https://doi.org/10.1111/coa.13417

41. Escera C, López-Caballero F, Gorina-Careta N. The potential effect of forbrain as an altered auditory feedback device. J Speech, Lang Hear Res [Internet]. 2018 Apr 1 [cited 2021 Mar 9];61(4):801-10. doi: https://doi.org/10.1044/2017_JSLHR-S-17-0072

42. Li Y, Tan M, Fan H, Wang EQ, Chen L, Li J, et al. Neurobehavioral Effects of LSVT® LOUD on Auditory-Vocal Integration in Parkinson's Disease: A Preliminary Study. Front Neurosci [nternet]. 2021 Feb 26;15. doi: https://doi.org/10.3389/fnins.2021.624801

43. Ramig LO, Gountryman S, O'Brien C, Hoehn M, Thompson L. Intensive speech treatment for patients with Parkinson's disease: Short- and long-term comparison of two techniques. Neurology [Internet]. 1996 Dec 1;47(6):1496-504. doi: https://doi.org/10.1212/ WNL.47.6.1496

44. Narayana S, Fox PT, Zhang W, Franklin C, Robin DA, Vogel D, et al. Neural correlates of efficacy of voice therapy in Parkinson's disease identified by performance-correlation analysis. Hum Brain Mapp [Internet]. 2010;31:222-236. doi: https://doi.org/10.1002/ hbm.20859

45. Segawa JA, Tourville JA, Beal DS, Guenther FH. The Neural Correlates of Speech Motor Sequence Learning. J Cogn Neurosci [Internet]. 2015 Apr;27(4):819-31. doi: https:// doi.org/10.1162/jocn_a_00737 